\title{
ON CLASSIFICATION OF PERMUTATIONS
}

\author{
FANCY NYABATE ${ }^{1}$, DENIS N. KING'ANG' ${ }^{2}$, SAMMY W. MUSUNDI ${ }^{1, *}$ \\ ${ }^{1}$ Deparment of Physical Sciences, Chuka University, Kenya \\ ${ }^{2}$ Department of Mathematics and Computer Science, University of Eldoret, Kenya \\ Corresponding author: sammusundi@yahoo.com
}

Received Mar. 10, 2020

\begin{abstract}
Aвstract. Some researchers in combinatorics have developed permutation algorithms using different approaches. We contribute to this area by developing a formula for generating permutations whereby, starting with an identity permutation, each succeeding permutation is a composition on the preceding one. We also determine the conditions by which the resulting permutations form a group.
\end{abstract}

2010 Mathematics Subject Classification. 20B05, 20B35, 20B30, $20 B 40$.

Key words and phrases. permutation; permutation algorithms; group.

\section{InTRODUCTION}

In this paper, we develop an algorithm for generating permutations whereby the initial permutation is the identity permutation and a composition rule is derived for computing the succeeding permutations. In the second section, we review permutations and show that the set of all permutations is a group under permutation multiplication. In section three, we present some developed permutation algorithms while in section four we present our results on permutation algorithm. In section five we give some algebraic properties of the generated permutations.

\section{Permutations}

A bijection is a mapping that is both one-to-one (hereby called an injection) and onto (hereby called a surjection). A permutation of a finite set $X$ is a function $\alpha: X \rightarrow X$ such that $\alpha$ is a 
bijection. For example, if $X=\{1,2,3\}$, then a mapping $\alpha: X \rightarrow X$ such that $1 \alpha=3,2 \alpha=1$ and $3 \alpha=2$ is a permutation of $X$, and is written as:

$$
\alpha=\left(\begin{array}{lll}
1 & 2 & 3 \\
3 & 1 & 2
\end{array}\right), \text { or simply } \alpha=\left(\begin{array}{lll}
3 & 1 & 2
\end{array}\right)
$$

In lemma 2.1, we show that composition of two permutations is a binary operation.

Lemma 2.1 Let $X$ be a finite set and let $\alpha_{i}, \alpha_{j}$ be any two permutations on $X$. Then their composition, written as $\alpha_{i} \alpha_{j}$, is another permutation on $X$.

\section{Proof}

Indeed, $\alpha_{i} \alpha_{j}$ is an injection, for if $x_{1}\left(\alpha_{i} \alpha_{j}\right)=x_{2}\left(\alpha_{i} \alpha_{j}\right)$, that is, if $\left(x_{1} \alpha_{i}\right) \alpha_{j}=\left(x_{2} \alpha_{i}\right) \alpha_{j}$, then $x_{1} \alpha_{i}=x_{2} \alpha_{i}$ (since $\alpha_{j}$ is one-to-one). But this implies that $x_{1}=x_{2}$ since $\alpha_{i}$ is also a bijection. Next, $\alpha_{i} \alpha_{j}$ is a surjection since whenever $x_{3} \in X$, then there must exist an $x_{2} \in X$ for which $x_{2} \alpha_{j}=x_{3}$ (since $\alpha_{j}$ is onto $X$ ). Also, since $\alpha_{i}$ is onto $X$, then there exists an $x_{1} \in X$ such that $x_{1} \alpha_{i}=x_{2}$. Thus, $x_{2} \alpha_{j}=\left(x_{1} \alpha_{i}\right) \alpha_{j}=x_{3}$, or $x_{1}\left(\alpha_{i} \alpha_{j}\right)=x_{3}$, and therefore, whenever $x_{3} \in X$ there must exist an $x_{1} \in X$ for which $x_{1}\left(\alpha_{i} \alpha_{j}\right)=x_{3}$, and so $\alpha_{i} \alpha_{j}$ is onto. Since $\alpha_{i} \alpha_{j}$ is both one-to-one and onto, we then conclude that it is a bijection and, consequently, is a permutation on $X$.

The binary operation in the above lemma is called permutation multiplication. As an example, if

$$
\alpha_{i}=\left(\begin{array}{lll}
1 & 2 & 3 \\
2 & 3 & 1
\end{array}\right) \text { and } \alpha_{j}=\left(\begin{array}{ccc}
1 & 2 & 3 \\
3 & 2 & 1
\end{array}\right)
$$

are two permutations on $X$, then $\alpha_{i} \alpha_{j}$ is the permutation obtained as follows:

$$
\alpha_{i} \alpha_{j}=\left(\begin{array}{lll}
1 & 2 & 3 \\
2 & 3 & 1
\end{array}\right)\left(\begin{array}{lll}
1 & 2 & 3 \\
3 & 2 & 1
\end{array}\right)=\left(\begin{array}{lll}
1 & 2 & 3 \\
2 & 1 & 3
\end{array}\right) .
$$

If $X$ has $n$ elements, then there are $n$ ! unique permutations on $X$.

In theorem 2.2, it is shown that the set of all permutations on a set $X$ is a group under permutation multiplication.

Theorem 2.2 [1]. Let $X$ be a non-empty set. Then the collection of all permutations on $X$ is a group under permutation multiplication.

\section{Proof}

We have seen in lemma 2.1 that permutation multiplication is a binary operation. Thus, if we denote the set of all permutations on $X$ by $\mathbf{P}$, then $\mathbf{P}$ is closed under this operation.

Now, permutation multiplication is defined as function composition, which is known to be associative. Next, there is a permutation $\alpha_{0} \in \mathbf{P}$ for which $x \alpha_{0}=x$ for all $x \in X$. $\alpha_{0}$ acts as the 
identity in $\mathbf{P}$. Finally, for a permutation $\alpha_{i} \in \mathbf{P}$, the inverse function $\alpha_{i}^{-1}$ is the permutation that reverses the direction of the mapping $\alpha_{i}$. Thus, $x \alpha_{i}^{-1}$ is the element $x^{\prime}$ of $X$ for which $x^{\prime} \alpha_{i}=x$. The existence of exactly one such an element $x^{i}$ is a consequence of the fact that, as a function, $\alpha_{i}^{-1}$ is a bijection. Thus, every member of $\mathbf{P}$ has an inverse in $\mathbf{P}$. Since $\mathbf{P}$ satisfies all the group axioms, we then conclude that it is a group.

The above group is called the symmetric group on $X$ and we shall denote it by $S_{n}$. This group is non-abelian.

In the ongoing example with $X=\{1,2,3\}, S_{3}$ has $3 !=6$ members. These are given below: $\alpha_{0}=\left(\begin{array}{lll}1 & 2 & 3 \\ 1 & 2 & 3\end{array}\right) ; \alpha_{1}=\left(\begin{array}{lll}1 & 2 & 3 \\ 2 & 3 & 1\end{array}\right) ; \alpha_{2}=\left(\begin{array}{lll}1 & 2 & 3 \\ 3 & 1 & 2\end{array}\right) ;$ $\alpha_{3}=\left(\begin{array}{lll}1 & 3 & 2 \\ 3 & 2 & 1\end{array}\right) ; \alpha_{4}=\left(\begin{array}{lll}1 & 2 & 3 \\ 3 & 2 & 1\end{array}\right) ; \alpha_{5}=\left(\begin{array}{lll}1 & 2 & 3 \\ 2 & 1 & 3\end{array}\right)$.

Thus, $\left(S_{3}, \circ\right)$ is a group where $S_{3}=\left\{\alpha_{0}, \alpha_{1}, \alpha_{2}, \alpha_{3}, \alpha_{4}, \alpha_{5}\right\}$ with $\alpha_{0}$ as the identity permutation and $\circ$ denote the permutation multiplication. This group is non-abelian. The group table for $\left(S_{3}, \circ\right)$ is given below:

TABle 1. $\left(S_{3}\right.$, o) Group Table

\begin{tabular}{c|cccccc}
$\circ$ & $\alpha_{0}$ & $\alpha_{1}$ & $\alpha_{2}$ & $\alpha_{3}$ & $\alpha_{4}$ & $\alpha_{5}$ \\
\hline$\alpha_{0}$ & $\alpha_{0}$ & $\alpha_{1}$ & $\alpha_{2}$ & $\alpha_{3}$ & $\alpha_{4}$ & $\alpha_{5}$ \\
$\alpha_{1}$ & $\alpha_{1}$ & $\alpha_{2}$ & $\alpha_{0}$ & $\alpha_{4}$ & $\alpha_{5}$ & $\alpha_{3}$ \\
$\alpha_{2}$ & $\alpha_{2}$ & $\alpha_{0}$ & $\alpha_{1}$ & $\alpha_{5}$ & $\alpha_{3}$ & $\alpha_{4}$ \\
$\alpha_{3}$ & $\alpha_{3}$ & $\alpha_{5}$ & $\alpha_{4}$ & $\alpha_{0}$ & $\alpha_{2}$ & $\alpha_{1}$ \\
$\alpha_{4}$ & $\alpha_{4}$ & $\alpha_{3}$ & $\alpha_{5}$ & $\alpha_{1}$ & $\alpha_{0}$ & $\alpha_{2}$ \\
$\alpha_{5}$ & $\alpha_{5}$ & $\alpha_{4}$ & $\alpha_{3}$ & $\alpha_{2}$ & $\alpha_{1}$ & $\alpha_{0}$
\end{tabular}

Theorem 2.3 [1]. Every group is isomorphic to a group of permutations.

A relation between non-empty sets $E$ and $F$ is a subset $R$ of $E \times F$. We read $(e, f) \in R$ as " $e$ is related to $f^{\prime \prime}$ and write $e R f$.

If $X$ is a non-empty set, an equivalence relation on $X$ is a subset $\sim$ of $X \times X$ which satisfies the following properties for all $x, y, z \in X$ :

i. Reflexive: $x \sim x$

ii. Symmetry: $x \sim y \Longrightarrow y \sim x$

iii. Transitive: $x \sim y$ and $y \sim z \Longrightarrow x \sim z$ 
An example of equivalence relation is the congruence modulo $n$ for a positive integer $n$. If $n \in \mathbb{N}^{+}$, define a relation $\sim: \mathbb{Z} \times \mathbb{Z} \Longrightarrow \mathbb{Z}$ by $h \sim k(\bmod n)$ if and only if $h-k=n t$ for some $t \in \mathbb{Z}$. Then $h \sim h(\bmod n)$ since $h-h=n(0)$ and $0 \in \mathbb{Z}$. Thus $\sim$ is reflexive. Next, if $h \sim k(\bmod n)$, then $h-k=n t$ for some $t \in \mathbb{Z}$. But this shows that $k-h=n(-t)$ and $-t \in \mathbb{Z}$ whenever $t \in \mathbb{Z}$. So $k \sim h(\bmod n)$ and this shows that $\sim$ is symmetric. Finally, let $h \sim k(\operatorname{modn})$ and $k \sim l(\bmod n)$. Then $h-k=n s$ and $k-l=n t$ for some $s, t \in \mathbb{Z}$. Adding the two equations we obtain $h-l=n(s+t)$ and $s+t \in \mathbb{Z}$ whenever $s, t \in \mathbb{Z}$. Therefore, $h \sim l(\bmod n)$ and this shows that $\sim$ is transitive. So we conclude that $\sim$ is an equivalence relation on $\mathbb{Z}$.

The equivalence relation in the above example is denoted by " $\equiv "$, that is, $h \equiv k(\bmod n)$ if and only if $h-k=n t$ for some $t \in \mathbb{Z}$. It is employed in developing a permutation algorithm in section four.

Each permutation $\alpha$ on a set $X$ determines a natural partition of $X$ into cells with the property that $x_{i}, x_{j} \in X$ are in the same cell if and only if $x_{j}=x_{i} \alpha^{n}$ for some $n \in \mathbb{Z}$. This partition is established by the following equivalence relation:

For each $x_{i}, x_{j} \in X$, let $x_{i} \sim x_{j}$ if and only if $x_{j}=x_{i} \alpha^{n}$ for some $n \in \mathbb{Z}$. Then $\sim$ is clearly an equivalence relation on $X$. Indeed, we have;

Reflexivity: $x_{i} \sim x_{i}$ since $x_{i}=x_{i} I=x_{i} \alpha^{0}$

Symmetry: If $x_{i} \sim x_{j}$, then $x_{j}=x_{i} \alpha^{n}$. But this implies that $x_{i}=x_{j} \alpha^{-n}$ and $-n \in \mathbb{Z}$ whenever $n \in \mathbb{Z}$. Thus, $x_{j} \sim x_{i}$.

Transitivity: Suppose that $x_{i} \sim x_{j}$ and $x_{j} \sim x_{k}$. Then $x_{i}=x_{j} \alpha^{n}$ and $x_{j}=x_{k} \alpha^{m}$ for some $m, n \in \mathbb{Z}$. Therefore, $x_{i}=\left(x_{k} \alpha^{n}\right) \alpha^{m}=x_{k} \alpha^{m+n}$ and $m+n \in \mathbb{Z}$ whenever $m, n \in \mathbb{Z}$. Thus, $x_{i} \sim x_{k}$.

If $\alpha$ is a permutation of $X$, then the equivalence classes in $X$ determined by the above equivalence relation are the orbits of $\alpha$.

A permutation $\alpha \in S_{n}$ is a cycle if it has at most one orbit containing more than one element. The length of a cycle is the number of elements in its orbit. A cycle of length 2 is called a transposition.

Theorems 2.4, 2.5 and 2.6 below give some properties of permutations.

Theorem 2.4 [1]. Every permutation of a non-empty set is a product of disjoint cycles.

Corollary 2.5 [1]. Any permutation of a finite set of at least two elements is a product of transpositions. 
Theorem 2.6 [1]. No permutation in $S_{n}$ can be expressed both as a product of an even number of transpositions and as a product of an odd number of transpositions.

A permutation of a finite set is even or odd according to whether it can be expressed as a product of an even number of transpositions or a product of an odd number of transpositions respectively.

Theorem 2.7 [1]. If $n \geq 2$ is the number of elements in $X$, then the group of all even permutations of $X$ is a subgroup of $S_{n}$ of order $\frac{n !}{2}$.

The subgroup of $S_{n}$ described in theorem 2.7 is called the alternating group on $X$ and is denoted by $A_{n}$.

\section{Permutations Algorithms}

Let $Q=\left\{q_{1}, q_{2}, \ldots, q_{n-1}\right\}$ be a sequence such that $q_{i}=+1$ or $q_{i}=-1$. A permutation with signature $Q$ is a permutation $\alpha=x_{1}, x_{2}, \ldots, x_{n}$ of the integers $1,2, \ldots, n$ such that $x_{i}<x_{i+1}$ if $q_{1}=+1$ or $x_{i}>x_{i+1}$ if $q_{1}=-1$ for all $i=1,2, \ldots, n-1$. An alternating permutation is a permutation with signature $Q=\left(+1,-1, \ldots,(-1)^{i+1}, \ldots\right)$.

Some researchers have worked on the problem of enumerating permutations with a given signature. The problem of generating all permutations with a given signature is equivalent to the problem of generating all topological sorting of a poset whose Hasse diagram is a path.

D. Roulants and F. Ruskey [2] in 1992 presented the first constant average time algorithm for generating all permutations with a given signature.

G. Sypro [3] in 2001 presented a new derivation of an enumeration formula for permutations of a given signature. They used random number sequences which mimic the permutations, in the sense that they rise and fall as determined by the permutation's signature.

A permutation $\alpha=(1 \alpha, 2 \alpha, \ldots, n \alpha)$ of $n$ integers $1,2, \ldots, n$ is said to have a rising sequence if $i \alpha<(i+1) \alpha<\ldots<(i+k) \alpha$. A falling sequence is defined similarly.

H. O. Foulkes [4], in 1970 described a method by which the enumeration of permutations of $1,2, \ldots, n$ with a prescribed sequence $A$ of rises and falls, or a prescribed sequence $B$ of inversions of orders, or with both $A$ and $B$, is effected in terms of numbers derived from the representation theory of the symmetric group.

N. Dershowitz [5], in 1975 presented an algorithm for generating permutations that generate the next permutation by reversing a certain suffix of its predecessor. A large number of generating functions for permutation statistics can be obtained by applying homomorphisms 
to simple symmetric function identities. In particular, a large number of generating functions involving the number of descents of a permutation $\alpha$, denoted $\operatorname{des}(\alpha)$, arises in this way.

F. Breuti [6],in 2000 introduced and studied a class of symmetric functions that depend on a parameter $q$, which include symmetric functions.

For a given finite set A of positive integers, J. Rammel and M. Riehl [7] in 2010 developed a method to produce similar generating functions for the set of permutations of the symmetric group $A_{n}$ whose descent set contains $A$.

The literature in this section motivates us to develop a permutation generating algorithm as a way of contributing to this topic. Our results are contained in sections four and five.

\section{Development of $\cup_{x_{k}}^{n}$-Permutations}

In this section, a class of permutations is obtained by first coming up with a mapping on a finite set. It is then proved that this mapping is a bijection and hence it is a permutation. Some examples of permutations are then provided.

Let $N$ be a finite set of ordered objects, say $N=\left\{x_{1}, x_{2}, x_{3}, \ldots, x_{n}\right\}$ for some $n \in \mathbb{N}$. Fix $\alpha_{0}=\left(\begin{array}{lllll}x_{1} & x_{2} & x_{3} & \ldots & x_{n}\end{array}\right)$. For some $q \in \mathbb{N}$ and such that $q$ divides $n$, partition $N$ into $q$ cells, that is, $C_{1}, C_{2}, \ldots, C_{q}$, whereby $x_{i} \in C_{j}$ if and only if $i \equiv j(\bmod q)$. Let the partition of $N$ allocates members of $N$ into the cells $C_{j}$ for $j=1,2, \ldots, q$ in a manner that their relative positions in $N$ is preserved.Therefore, we obtain a partition $P_{\alpha_{0}}^{n}=\left\{C_{j}: x_{i} \in C_{j}\right.$ if and only if $\left.i \equiv j(\operatorname{modq})\right\}$

For a fixed $x_{k} \in N$, the union $\bigcup_{x_{k}}^{n}$ of the cells in $P_{\alpha_{0}}^{n}$ is obtained as follows:

$\bigcup_{x_{k}}^{n}\left[P_{\alpha_{0}}^{n}\right]=\bigcup_{j=1}^{q}\left\{C_{j}: C_{r}\right.$ is at fixed position $t$ if $\left.x_{k} \in C_{r}\right\}$, where $t=1,2, \ldots, q$. Again, the union of the cells $C_{j}$ for $j=1,2, \ldots, q$ are such that the relative positions of the cells in $P_{\alpha_{0}}^{n}$ are preserved.

Let $\bigcup_{x_{k}}^{n}\left[P_{\alpha_{0}}^{n}\right]=\alpha_{1}$. Repeat the above process in succession to obtain $\alpha_{2}, \alpha_{3} \ldots$. etc. We thus obtain a mapping $\bigcup_{x_{k}}^{n}: N \rightarrow N$ such that:

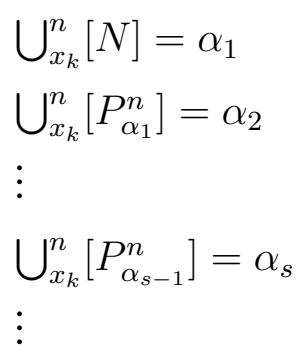

Theorem 4.1 The mapping $\bigcup_{x_{k}}^{n}: N \rightarrow N$ described above is a permutation in $N$.

\section{Proof}


We need to show that $\bigcup_{x_{k}}^{n}$ is a bijection on $N$. First, $\bigcup_{x_{k}}^{n}$ is one-to-one for if $x_{i}, x_{j} \in N$ with $\bigcup_{x_{k}}^{n}\left(x_{i}\right)=\bigcup_{x_{k}}^{n}\left(x_{j}\right)$, then $x_{i}=x_{j}$ necessarily for there are no instances where distinct members of $N$ are mapped to identical members of $N$ under $\bigcup_{x_{k}}^{n}$. Thus $\bigcup_{x_{k}}^{n}$ is one-to-one.

Next, $\bigcup_{x_{k}}^{n}$ is onto $N$ since every member of $N$ is some $\bigcup_{x_{k}}^{n}$-image of a member in $N$. Therefore, we conclude that $\bigcup_{x_{k}}^{n}$ is a permutation on $N$.

Notation 4.2: We shall denote the set of all such permutations on $N$ by $N_{x_{k}}^{n}$ and call them the $\bigcup_{x_{k}}^{n}$-permutations.

Corollary 4.3 The set of all $\bigcup_{x_{k}}^{n}$-permutations on $N$ is a subset of $N_{n}$, the set of all permutations on $N$.

\section{Proof}

Now, from theorem 4.1, if $\alpha \in N_{x_{k}}^{n}$ then $\alpha$ is a permutation on $N$ and hence $\alpha \in N_{n}$, the set of all permutations on $N$.

Three categories of permutations are considered for construction, that is, when $n=9, n=12$ and $n=15$. To obtain the first category of permutations, we consider $q=3, k=4 n=9$ and $t$ is the middle position.

Let $N=\{1,2,3,4,5,6,7,8,9\}$. Note that here $x_{k}=x_{4}=4$. Then

$$
\alpha_{0}=\left(\begin{array}{lllllllll}
1 & 2 & 3 & 4 & 5 & 6 & 7 & 8 & 9
\end{array}\right)
$$

Therefore,

$$
\alpha_{0}=\left(\begin{array}{ccccccccc}
1 & 2 & 3 & 4 & 5 & 6 & 7 & 8 & 9 \\
1 & 2 & 3 & 4 & 5 & 6 & 7 & 8 & 9
\end{array}\right)
$$

When $N$ is mapped the first time, the first permutation denoted by $\alpha_{1}$ is obtained as shown below:

$$
\begin{aligned}
\alpha_{0} & =\left(\begin{array}{lllllllll}
1 & 2 & 3 & 4 & 5 & 6 & 7 & 8 & 9
\end{array}\right) \\
C_{1} & =\{1,4,7\}, C_{2}=\{2,5,8\}, C_{3}=\{3,6,9\}
\end{aligned}
$$

Then,

$$
P_{\alpha_{0}}^{9}=\{\{1,4,7\},\{2,5,8\},\{3,6,9\}\}
$$

Taking the union,we obtain

$$
\bigcup_{4}^{9}\left[P_{\alpha_{0}}^{9}\right]=\{3,6,9,1,4,7,2,5,8\}
$$

Therefore

$$
\alpha_{1}=\left(\begin{array}{lllllllll}
1 & 2 & 3 & 4 & 5 & 6 & 7 & 8 & 9 \\
3 & 6 & 9 & 1 & 4 & 7 & 2 & 5 & 8
\end{array}\right)
$$

A mapping of $\alpha_{1}$ yields the second permutation denoted by $\alpha_{2}$. This is possible after partitioning it into three cells as follows: 


$$
\begin{aligned}
& \alpha_{1}=\left(\begin{array}{lllllllll}
3 & 6 & 9 & 1 & 4 & 7 & 2 & 5 & 8
\end{array}\right) \\
& C_{1}=\{3,1,2\}, C_{2}=\{6,4,5\}, C_{3}=\{9,7,8\}
\end{aligned}
$$

Then,

$$
P_{\alpha_{1}}^{9}=\{\{3,1,2\},\{6,4,5\},\{9,7,8\}\}
$$

Taking the union, we obtain

$$
\bigcup_{4}^{9}\left[P_{\alpha_{1}}^{9}\right]=\{3,1,2,6,4,5,9,7,8\}
$$

Therefore

$$
\alpha_{2}=\left(\begin{array}{lllllllll}
1 & 2 & 3 & 4 & 5 & 6 & 7 & 8 & 9 \\
3 & 1 & 2 & 6 & 4 & 5 & 9 & 7 & 8
\end{array}\right)
$$

From the first category, we obtain three unique permutations, that is;

$$
N_{4}^{9}=\left\{\alpha_{0}, \alpha_{1}, \alpha_{2}\right\} \text {. }
$$

Constructing the second category of permutations, we consider $n=12, k=4, q=3, t$ is the middle position, and $N=\{1,2,3,4,5,6,7,8,9,10,11,12\}$. Again, $x_{k}=x_{4}=4$. Since $N=\{1,2,3,4,5,6,7,8,9,10,11,12\}$, then we have

$$
\alpha_{0}=\left(\begin{array}{llllllllllll}
1 & 2 & 3 & 4 & 5 & 6 & 7 & 8 & 9 & 10 & 11 & 12 \\
1 & 2 & 3 & 4 & 5 & 6 & 7 & 8 & 9 & 10 & 11 & 12
\end{array}\right)
$$

When $N$ is mapped for the first time, the first permutation denoted by $\alpha_{1}$ is obtained as follows;

$$
\begin{aligned}
& \alpha_{0}=\left(\begin{array}{llllllllllll}
1 & 2 & 3 & 4 & 5 & 6 & 7 & 8 & 9 & 10 & 11 & 12
\end{array}\right) \\
& C_{1}=\{1,4,7,10\}, C_{2}=\{2,5,8,11\}, C_{3}=\{3,6,9,12\}
\end{aligned}
$$

Then,

$$
P_{\alpha_{0}}^{12}=\{\{1,4,7,10\},\{2,5,8,11\},\{3,6,9,12\}\}
$$

Taking the union, we obtain

$$
\bigcup_{4}^{12}\left[P_{\alpha_{0}}^{12}\right]=\{3,6,9,12,1,4,7,10,2,5,8,11\}
$$

Therefore

$$
\alpha_{1}=\left\{\begin{array}{cccccccccccc}
1 & 2 & 3 & 4 & 5 & 6 & 7 & 8 & 9 & 10 & 11 & 12 \\
3 & 6 & 9 & 12 & 1 & 4 & 7 & 10 & 2 & 5 & 8 & 11
\end{array}\right\}
$$

The next permutation denoted by $\alpha_{2}$ is obtained when $N$ undergoes the second permutation as follows;

$$
\begin{aligned}
& \alpha_{1}=\left(\begin{array}{llllllllllll}
3 & 6 & 9 & 12 & 1 & 4 & 7 & 10 & 2 & 5 & 8 & 11
\end{array}\right) \\
& C_{1}=\{3,12,7,5\}, C_{2}=\{6,1,10,8\}, C_{3}=\{9,4,2,11\}
\end{aligned}
$$

Then,

$$
P_{\alpha_{1}}^{12}=\{\{3,12,7,5\},\{6,1,10,8\},\{9,4,2,11\}\}
$$

Taking the union, we obtain 


$$
\bigcup_{4}^{12}\left[P_{\alpha_{1}}^{12}\right]=\{6,1,10,8,9,4,2,11,3,12,7,5\}
$$

Therefore

$$
\alpha_{2}=\left(\begin{array}{cccccccccccc}
1 & 2 & 3 & 4 & 5 & 6 & 7 & 8 & 9 & 10 & 11 & 12 \\
6 & 1 & 10 & 8 & 9 & 4 & 2 & 11 & 3 & 12 & 7 & 5
\end{array}\right)
$$

When $N$ undergoes the next permutation, we obtain $\alpha_{3}$ as follows:

$$
\begin{aligned}
\alpha_{2} & =\left(\begin{array}{llllllllllll}
6 & 1 & 10 & 8 & 9 & 4 & 2 & 11 & 3 & 12 & 7 & 5
\end{array}\right) \\
C_{1} & =\{6,8,2,12\}, C_{2}=\{1,9,11,7\}, C_{3}=\{10,4,3,5\}
\end{aligned}
$$

Then,

$$
P_{\alpha_{2}}^{12}=\{\{6,8,2,12\},\{1,9,11,7\},\{10,4,3,5\}\}
$$

Taking the union,we obtain

$$
\bigcup_{4}^{12}\left[P_{\alpha_{2}}^{12}\right]=\{1,9,11,7,10,4,3,5,6,8,2,12\}
$$

Therefore

$$
\alpha_{3}=\left(\begin{array}{cccccccccccc}
1 & 2 & 3 & 4 & 5 & 6 & 7 & 8 & 9 & 10 & 11 & 12 \\
1 & 9 & 11 & 7 & 10 & 4 & 3 & 5 & 6 & 8 & 2 & 12
\end{array}\right)
$$

The fourth permutation is obtained as follows:

$$
\begin{aligned}
\alpha_{3} & =\left(\begin{array}{llllllllllll}
1 & 9 & 11 & 7 & 10 & 4 & 3 & 5 & 6 & 8 & 2 & 12
\end{array}\right) \\
C_{1} & =\{1,7,3,8\}, C_{2}=\{9,10,5,2\}, C_{3}=\{11,4,6,12\}
\end{aligned}
$$

Then,

$$
P_{\alpha_{3}}^{12}=\{\{1,7,3,8\},\{9,10,5,2\},\{11,4,6,12\}\}
$$

Taking the union,we obtain

$$
\bigcup_{4}^{12}\left[P_{\alpha_{3}}^{12}\right]=\{9,10,5,2,11,4,6,12,1,7,3,8\}
$$

Therefore

$$
\alpha_{4}=\left(\begin{array}{cccccccccccc}
1 & 2 & 3 & 4 & 5 & 6 & 7 & 8 & 9 & 10 & 11 & 12 \\
9 & 10 & 5 & 2 & 11 & 4 & 6 & 12 & 1 & 7 & 3 & 8
\end{array}\right)
$$

When $N$ undergoes the fifth permutation, we obtain $\alpha_{5}$ as follows;

$$
\begin{aligned}
\alpha_{4} & =\left(\begin{array}{llllllllllll}
9 & 10 & 5 & 2 & 11 & 4 & 6 & 12 & 1 & 7 & 3 & 8
\end{array}\right) \\
C_{1} & =\{9,2,6,7\}, C_{2}=\{10,11,12,3\}, C_{3}=\{5,4,1,8\}
\end{aligned}
$$

Then,

$$
P_{\alpha_{4}}^{12}=\{\{9,2,6,7\},\{10,11,12,3\},\{5,4,1,8\}\}
$$

Taking the union,we obtain

$$
\bigcup_{4}^{12}\left[P_{\alpha_{4}}^{12}\right]=\{10,11,12,3,5,4,1,8,9,2,6,7\}
$$

Therefore

$$
\alpha_{5}=\left(\begin{array}{cccccccccccc}
1 & 2 & 3 & 4 & 5 & 6 & 7 & 8 & 9 & 10 & 11 & 12 \\
10 & 11 & 12 & 3 & 5 & 4 & 1 & 8 & 9 & 2 & 6 & 7
\end{array}\right)
$$


When $N$ is mapped for the sixth time, the sixth permutation denoted by $\alpha_{6}$ is obtained as follows;

$$
\begin{aligned}
\alpha_{5} & =\left(\begin{array}{llllllllllll}
10 & 11 & 12 & 3 & 5 & 4 & 1 & 8 & 9 & 2 & 6 & 7
\end{array}\right) \\
C_{1} & =\{10,3,1,2\}, C_{2}=\{11,5,8,6\}, C_{3}=\{12,4,9,7\}
\end{aligned}
$$

Then,

$$
P_{\alpha_{5}}^{12}=\{\{10,3,1,2\},\{11,5,8,6\},\{12,4,9,7\}\}
$$

Taking the union, we obtain

$$
\bigcup_{4}^{12}\left[P_{\alpha_{5}}^{12}\right]=\{11,5,8,6,12,4,9,7,10,3,1,2\}
$$

Therefore

$$
\alpha_{6}=\left\{\begin{array}{cccccccccccc}
1 & 2 & 3 & 4 & 5 & 6 & 7 & 8 & 9 & 10 & 11 & 12 \\
11 & 5 & 8 & 6 & 12 & 4 & 9 & 7 & 10 & 3 & 1 & 2
\end{array}\right\}
$$

The next permutation denoted by $\alpha_{7}$ is obtained when $N$ undergoes the seventh permutation as follows;

$$
\begin{aligned}
\alpha_{6} & =\left(\begin{array}{llllllllllll}
11 & 5 & 8 & 6 & 12 & 4 & 9 & 7 & 10 & 3 & 1 & 2
\end{array}\right) \\
C_{1} & =\{11,6,9,3\}, C_{2}=\{5,12,7,1\}, C_{3}=\{8,4,10,2\}
\end{aligned}
$$

Then,

$$
P_{\alpha_{6}}^{12}=\{\{11,6,9,3\},\{5,12,7,1\},\{8,4,10,2\}\}
$$

Taking the union, we obtain

$$
\bigcup_{4}^{12}\left[P_{\alpha_{6}}^{12}\right]=\{5,12,7,1,8,4,10,2,11,6,9,3\}
$$

Therefore

$$
\alpha_{7}=\left(\begin{array}{cccccccccccc}
1 & 2 & 3 & 4 & 5 & 6 & 7 & 8 & 9 & 10 & 11 & 12 \\
5 & 12 & 7 & 1 & 8 & 4 & 10 & 2 & 11 & 6 & 9 & 3
\end{array}\right)
$$

When $N$ undergoes the next permutation, we obtain $\alpha_{8}$ as follows;

$$
\begin{aligned}
& \alpha_{7}=\left(\begin{array}{llllllllllll}
5 & 12 & 7 & 1 & 8 & 4 & 10 & 2 & 11 & 6 & 9 & 3
\end{array}\right) \\
& C_{1}=\{5,1,10,6\}, C_{2}=\{12,8,2,9\}, C_{3}=\{7,4,11,3\} \text { Then, } \\
& P_{\alpha_{7}}^{12}=\{\{5,1,10,6\},\{12,8,2,9\},\{7,4,11,3\}\}
\end{aligned}
$$

Taking the union,we obtain

$$
\bigcup_{4}^{12}\left[P_{\alpha_{7}}^{12}\right]=\{12,8,2,9,7,4,11,3,5,1,10,6\}
$$

Therefore

$$
\alpha_{8}=\left(\begin{array}{cccccccccccc}
1 & 2 & 3 & 4 & 5 & 6 & 7 & 8 & 9 & 10 & 11 & 12 \\
12 & 8 & 2 & 9 & 7 & 4 & 11 & 3 & 5 & 1 & 10 & 6
\end{array}\right)
$$

The ninth permutation is obtained as follows:

$$
\begin{aligned}
\alpha_{8} & =\left(\begin{array}{llllllllllll}
12 & 8 & 2 & 9 & 7 & 4 & 11 & 3 & 5 & 1 & 10 & 6
\end{array}\right) \\
C_{1} & \left.=\{12,9,11,1\}, C_{2}=\{8,7,3,10\}, C_{3}=\{2,4,5,6\}\right\}
\end{aligned}
$$


Then,

$$
P_{\alpha_{8}}^{12}=\{\{12,9,11,1\},\{8,7,3,10\},\{2,4,5,6\}
$$

Taking the union,we obtain

$$
\bigcup_{4}^{12}\left[P_{\alpha_{8}}^{12}\right]=\{8,7,3,10,2,4,5,6,12,9,11,1\}
$$

Therefore

$$
\alpha_{9}=\left(\begin{array}{cccccccccccc}
1 & 2 & 3 & 4 & 5 & 6 & 7 & 8 & 9 & 10 & 11 & 12 \\
8 & 7 & 3 & 10 & 2 & 4 & 5 & 6 & 12 & 9 & 1 & 1
\end{array}\right)
$$

When $N$ undergoes the tenth permutation, we obtain $\alpha_{10}$ as follows;

$$
\begin{aligned}
\alpha_{9} & =\left(\begin{array}{llllllllllll}
8 & 7 & 3 & 10 & 2 & 4 & 5 & 6 & 12 & 9 & 1 & 1
\end{array}\right) \\
C_{1} & =\{8,10,5,9\}, C_{2}=\{7,2,6,11\}, C_{3}=\{3,4,12,1\}
\end{aligned}
$$

Then,

$$
P_{\alpha_{9}}^{12}=\{\{8,10,5,9\},\{7,2,6,11\},\{3,4,12,1\}\}
$$

Taking the union,we obtain

$$
\bigcup_{4}^{12}\left[P_{\alpha_{9}}^{12}\right]=\{7,2,6,11,3,4,12,1,8,10,5,9\}
$$

Therefore

$$
\alpha_{10}=\left(\begin{array}{cccccccccccc}
1 & 2 & 3 & 4 & 5 & 6 & 7 & 8 & 9 & 10 & 11 & 12 \\
7 & 12 & 6 & 11 & 3 & 4 & 12 & 1 & 8 & 10 & 5 & 9
\end{array}\right)
$$

When $N$ undergoes the eleventh permutation, we obtain $\alpha_{11}$ as follows;

$$
\begin{aligned}
& \alpha_{10}=\left(\begin{array}{llllllllllll}
7 & 12 & 6 & 11 & 3 & 4 & 12 & 1 & 8 & 10 & 5 & 9
\end{array}\right) \\
& C_{1}=\{7,11,12,10\}, C_{2}=\{2,3,1,5\}, C_{3}=\{6,4,8,9\}
\end{aligned}
$$

Then,

$$
P_{\alpha_{10}}^{12}=\{\{7,11,12,10\},\{2,3,1,5\},\{6,4,8,9\}\}
$$

Taking the union,we obtain

$$
\bigcup_{4}^{12}\left[P_{\alpha_{10}}^{12}\right]=\{2,3,1,5,6,4,8,9,7,11,12,10\}
$$

Therefore

$$
\alpha_{11}=\left(\begin{array}{llllllllllll}
1 & 2 & 3 & 4 & 5 & 6 & 7 & 8 & 9 & 10 & 11 & 12 \\
2 & 3 & 1 & 5 & 6 & 4 & 8 & 9 & 7 & 11 & 12 & 10
\end{array}\right)
$$

From the second category of permutations, we obtain twelve unique permutations, that is, $N_{4}^{12}=\left\{\alpha_{0}, \alpha_{1}, \alpha_{2}, \alpha_{3}, \alpha_{4}, \alpha_{5}, \alpha_{6}, \alpha_{7}, \alpha_{8}, \alpha_{9}, \alpha_{10}, \alpha_{11}\right\}$.

The third category of permutations is obtained, when we consider $k=4, q=3, t$ is the middle position and

$$
N=\left\{\begin{array}{lllllllllllllll}
1 & 2 & 3 & 4 & 5 & 6 & 7 & 8 & 9 & 10 & 11 & 12 & 13 & 14 & 15
\end{array}\right\}
$$

Therefore, 


$$
\alpha_{0}=\left(\begin{array}{lllllllllllllll}
1 & 2 & 3 & 4 & 5 & 6 & 7 & 8 & 9 & 10 & 11 & 12 & 13 & 14 & 15 \\
1 & 2 & 3 & 4 & 5 & 6 & 7 & 8 & 9 & 10 & 11 & 12 & 13 & 14 & 15
\end{array}\right)
$$

When $N$ undergoes the first permutation, we obtain $\alpha_{1}$ as shown below:

$$
\begin{aligned}
& \alpha_{0}=\left(\begin{array}{lllllllllllllll}
1 & 2 & 3 & 4 & 5 & 6 & 7 & 8 & 9 & 10 & 11 & 12 & 13 & 14 & 15
\end{array}\right) \\
& C_{1}=\{1,4,7,10,13\}, C_{2}=\{2,5,8,11,14\}, C_{3}=\{3,6,9,12,15\}
\end{aligned}
$$

Then,

$$
P_{\alpha_{0}}^{15}=\{\{1,4,7,10,13\},\{2,5,8,11,14\},\{3,6,9,12,15\}\}
$$

Taking the union, we obtain

$$
\bigcup_{4}^{15}\left[P_{\alpha_{0}}^{15}\right]=\{3,6,9,12,15,1,4,7,10,13,2,5,8,11,14\}
$$

Therefore

$$
\alpha_{1}=\left(\begin{array}{ccccccccccccccc}
1 & 2 & 3 & 4 & 5 & 6 & 7 & 8 & 9 & 10 & 11 & 12 & 13 & 14 & 15 \\
3 & 6 & 9 & 12 & 15 & 1 & 4 & 7 & 10 & 13 & 2 & 5 & 8 & 11 & 14
\end{array}\right)
$$

When $N$ undergoes the next mapping we obtain permutation $\alpha_{2}$ as shown below;

$$
\begin{aligned}
& \alpha_{1}=\left(\begin{array}{lllllllllllllll}
3 & 6 & 9 & 12 & 15 & 1 & 4 & 7 & 10 & 13 & 2 & 5 & 8 & 11 & 14
\end{array}\right) \\
& C_{1}=\{3,12,4,13,8\}, C_{2}=\{6,15,7,2,11\}, C_{3}=\{9,1,10,5,14\}
\end{aligned}
$$

Then,

$$
P_{\alpha_{1}}^{15}=\{\{3,12,4,13,8\},\{6,15,7,2,11\},\{9,1,10,5,14\}\}
$$

Taking the union, we obtain

$$
\bigcup_{4}^{15}\left[P_{\alpha_{1}}^{15}\right]=\{9,1,10,5,14,3,12,4,13,8,6,15,7,2,11\}
$$

Therefore

$$
\alpha_{2}=\left(\begin{array}{ccccccccccccccc}
1 & 2 & 3 & 4 & 5 & 6 & 7 & 8 & 9 & 10 & 11 & 12 & 13 & 14 & 15 \\
9 & 1 & 10 & 5 & 14 & 3 & 12 & 4 & 13 & 8 & 6 & 15 & 7 & 2 & 11
\end{array}\right)
$$

$N$ undergoes the third permutation, we obtain $\alpha_{3}$ as shown below;

$$
\begin{aligned}
& \alpha_{2}=\left(\begin{array}{lllllllllllllll}
9 & 1 & 10 & 5 & 14 & 3 & 12 & 4 & 13 & 8 & 6 & 15 & 7 & 2 & 11
\end{array}\right) \\
& C_{1}=\{9,5,12,8,7\}, C_{2}=\{1,14,4,6,2\}, C_{3}=\{10,3,13,15,11\}
\end{aligned}
$$

Then,

$$
P_{\alpha_{2}}^{15}=\{\{9,5,12,8,7\},\{1,14,4,6,2\},\{10,3,13,15,11\}\}
$$

Taking the union, we obtain

$$
\bigcup_{4}^{15}\left[P_{\alpha_{2}}^{15}\right]=\{9,5,12,8,7,1,14,4,6,2,10,3,13,15,11\}
$$

Therefore

$$
\alpha_{3}=\left(\begin{array}{ccccccccccccccc}
1 & 2 & 3 & 4 & 5 & 6 & 7 & 8 & 9 & 10 & 11 & 12 & 13 & 14 & 15 \\
9 & 5 & 12 & 8 & 7 & 1 & 14 & 4 & 6 & 2 & 10 & 3 & 13 & 15 & 11
\end{array}\right)
$$

The fourth permutation denoted by $\alpha_{4}$ is obtained when $N$ undergoes the fourth permutation as shown below; 


$$
\begin{aligned}
& \alpha_{3}=\left(\begin{array}{lllllllllllllll}
9 & 5 & 12 & 8 & 7 & 1 & 14 & 4 & 6 & 2 & 10 & 3 & 13 & 15 & 11
\end{array}\right) \\
& C_{1}=\{9,8,14,2,13\}, C_{2}=\{5,7,4,10,15\}, C_{3}=\{12,1,6,3,11\}
\end{aligned}
$$

Then,

$$
P_{\alpha_{3}}^{15}=\{\{9,8,14,2,13\},\{5,7,4,10,15\},\{12,1,6,3,11\}\}
$$

Taking the union,we obtain

$$
\bigcup_{4}^{15}\left[P_{\alpha_{3}}^{15}\right]=\{9,8,14,2,13,5,7,4,10,15,12,1,6,3,11\}
$$

Therefore

$$
\alpha_{4}=\left(\begin{array}{ccccccccccccccc}
1 & 2 & 3 & 4 & 5 & 6 & 7 & 8 & 9 & 10 & 11 & 12 & 13 & 14 & 15 \\
9 & 8 & 14 & 2 & 13 & 5 & 7 & 4 & 10 & 15 & 12 & 1 & 6 & 3 & 11
\end{array}\right)
$$

When $N$ goes through the fifth permutation, we obtain $\alpha_{5}$ as shown below;

$$
\begin{aligned}
\alpha_{4} & =\left(\begin{array}{lllllllllllllll}
9 & 8 & 14 & 2 & 13 & 5 & 7 & 4 & 10 & 15 & 12 & 1 & 6 & 3 & 11
\end{array}\right) \\
C_{1} & =\{9,2,7,15,6\}, C_{2}=\{8,13,4,12,3\}, C_{3}=\{14,5,10,1,11\}
\end{aligned}
$$

Then,

$$
P_{\alpha_{4}}^{15}=\{\{9,2,7,15,6\},\{8,13,4,12,3\},\{14,5,10,1,11\}\}
$$

Taking the union,we obtain

$$
\bigcup_{4}^{15}\left[P_{\alpha_{4}}^{15}\right]=\{9,2,7,15,6,8,13,4,12,3,14,5,10,1,11\}
$$

Therefore

$$
\alpha_{5}=\left(\begin{array}{ccccccccccccccc}
1 & 2 & 3 & 4 & 5 & 6 & 7 & 8 & 9 & 10 & 11 & 12 & 13 & 14 & 15 \\
9 & 2 & 7 & 15 & 6 & 8 & 13 & 4 & 12 & 3 & 14 & 5 & 10 & 1 & 11
\end{array}\right)
$$

The sixth permutation $\alpha_{6}$ is obtained when the $N$ undergoes the sixth mapping as shown below;

$$
\begin{aligned}
& \alpha_{5}=\left(\begin{array}{lllllllllllllll}
9 & 2 & 7 & 15 & 6 & 8 & 13 & 4 & 12 & 3 & 14 & 5 & 10 & 1 & 11
\end{array}\right) \\
& C_{1}=\{9,15,13,3,10\}, C_{2}=\{2,6,4,14,1\}, C_{3}=\{7,8,12,5,11\}
\end{aligned}
$$

Then,

$$
P_{\alpha_{5}}^{15}=\{\{9,15,13,3,10\},\{2,6,4,14,1\},\{7,8,12,5,11\}\}
$$

Taking the union,we obtain

$$
\bigcup_{4}^{15}\left[P_{\alpha_{5}}^{15}\right]=\{9,15,13,3,10,2,6,4,14,1,7,8,12,5,11\}
$$

Therefore

$$
\alpha_{6}=\left(\begin{array}{ccccccccccccccc}
1 & 2 & 3 & 4 & 5 & 6 & 7 & 8 & 9 & 10 & 11 & 12 & 13 & 14 & 15 \\
9 & 15 & 13 & 3 & 10 & 2 & 6 & 4 & 14 & 1 & 7 & 8 & 12 & 5 & 11
\end{array}\right)
$$

The seventh permutation denoted by $\alpha_{7}$ is obtained when $N$ undergoes the seventh permutation as shown below;

$$
\begin{aligned}
& \alpha_{6}=\left(\begin{array}{lllllllllllllll}
9 & 15 & 13 & 3 & 10 & 2 & 6 & 4 & 14 & 1 & 7 & 8 & 12 & 5 & 11
\end{array}\right) \\
& C_{1}=\{9,3,6,1,12\}, C_{2}=\{15,10,4,7,5\}, C_{3}=\{13,2,14,8,11\}
\end{aligned}
$$


Then,

$$
P_{\alpha_{6}}^{15}=\{\{9,3,6,1,12\},\{15,10,4,7,5\},\{13,2,14,8,11\}\}
$$

Taking the union, we obtain

$$
\bigcup_{4}^{15}\left[P_{\alpha_{6}}^{15}\right]=\{9,3,6,1,12,15,10,4,7,5,13,2,14,8,11\}
$$

Therefore

$$
\alpha_{7}=\left(\begin{array}{ccccccccccccccc}
1 & 2 & 3 & 4 & 5 & 6 & 7 & 8 & 9 & 10 & 11 & 12 & 13 & 14 & 15 \\
9 & 3 & 6 & 1 & 12 & 15 & 10 & 4 & 7 & 5 & 13 & 2 & 14 & 8 & 11
\end{array}\right)
$$

From the third category of permutations, we obtain eight unique permutations, that is, $N_{4}^{15}=\left\{\alpha_{0}, \alpha_{1}, \alpha_{2}, \alpha_{3}, \alpha_{4}, \alpha_{5}, \alpha_{6}, \alpha_{7}\right\}$.

Three categories of permutations have been generated in this section. Using the equivalence relation introduced in section 2 we can present these permutations in terms of their cycles as shown below:

When $n=9$, the following permutations are obtained;

$$
\begin{aligned}
& \alpha_{0}=(1)(2)(3)(4)(5)(6)(7)(8)(9) \\
& \alpha_{1}=\left(\begin{array}{llllll}
1 & 3 & 9 & 8 & 5 & 4
\end{array}\right)\left(\begin{array}{lll}
2 & 6 & 7
\end{array}\right) \\
& \alpha_{2}=\left(\begin{array}{lll}
1 & 3 & 2
\end{array}\right)\left(\begin{array}{lll}
4 & 6 & 5
\end{array}\right)\left(\begin{array}{lll}
7 & 9 & 8
\end{array}\right)
\end{aligned}
$$

When $n=12$, we have 12 permutations whose cycles are as follows;

$$
\begin{aligned}
& \alpha_{0}=(1)(2)(3)(4)(5)(6)(7)(8)(9)(10)(11)(12) \\
& \alpha_{1}=\left(\begin{array}{lllllllllll}
1 & 3 & 9 & 2 & 6 & 4 & 12 & 11 & 8 & 10 & 5
\end{array}\right)\left(\begin{array}{l}
7 \\
1
\end{array}\right) \\
& \alpha_{2}=\left(\begin{array}{lllllll}
1 & 6 & 4 & 8 & 11 & 7 & 2
\end{array}\right)\left(\begin{array}{lllll}
3 & 10 & 12 & 5 & 9
\end{array}\right) \\
& \alpha_{3}=\left(\begin{array}{l}
1
\end{array}\right)\left(\begin{array}{lllllll}
2 & 9 & 6 & 4 & 7 & 3 & 11
\end{array}\right)\left(\begin{array}{lll}
5 & 10 & 8
\end{array}\right)\left(\begin{array}{l}
12
\end{array}\right) \\
& \alpha_{4}=\left(\begin{array}{ll}
1 & 9
\end{array}\right)\left(\begin{array}{lllll}
2 & 10 & 7 & 6 & 4
\end{array}\right)\left(\begin{array}{lll}
3 & 5 & 11
\end{array}\right)\left(\begin{array}{ll}
8 & 12
\end{array}\right) \\
& \alpha_{5}=\left(\begin{array}{llllll}
1 & 10 & 2 & 11 & 6 & 4
\end{array}\right)\left(\begin{array}{l}
5 \\
5
\end{array}\right)\left(\begin{array}{l}
8
\end{array}\right)(9) \\
& \alpha_{6}=\left(\begin{array}{ll}
1 & 11
\end{array}\right)\left(\begin{array}{lll}
2 & 5 & 12
\end{array}\right)\left(\begin{array}{lllll}
3 & 8 & 7 & 9 & 10
\end{array}\right)\left(\begin{array}{ll}
4 & 6
\end{array}\right) \\
& \alpha_{7}=\left(\begin{array}{llllllllll}
1 & 5 & 8 & 2 & 12 & 3 & 7 & 10 & 6 & 4
\end{array}\right)\left(\begin{array}{ll}
9 & 11
\end{array}\right) \\
& \alpha_{8}=\left(\begin{array}{lllllllll}
1 & 12 & 6 & 4 & 9 & 5 & 7 & 11 & 10
\end{array}\right)\left(\begin{array}{lll}
2 & 8 & 3
\end{array}\right) \\
& \alpha_{9}=\left(\begin{array}{lllllll}
1 & 8 & 6 & 4 & 10 & 9 & 12
\end{array}\right)\left(\begin{array}{lll}
2 & 7 & 5
\end{array}\right)\left(\begin{array}{l}
3
\end{array}\right)\left(\begin{array}{l}
11
\end{array}\right) \\
& \alpha_{10}=\left(\begin{array}{lllll}
1 & 7 & 12 & 9 & 8
\end{array}\right)\left(\begin{array}{l}
2
\end{array}\right)\left(\begin{array}{lllll}
3 & 6 & 4 & 11 & 5
\end{array}\right)\left(\begin{array}{l}
10
\end{array}\right) \\
& \alpha_{11}=\left(\begin{array}{lll}
1 & 2 & 3
\end{array}\right)\left(\begin{array}{lll}
4 & 5 & 6
\end{array}\right)\left(\begin{array}{lll}
7 & 8 & 9
\end{array}\right)\left(\begin{array}{lll}
10 & 11 & 12
\end{array}\right)
\end{aligned}
$$

When $n=15$, there are 8 permutations and their cycles are given below;

$$
\begin{aligned}
& \alpha_{0}=(1)(2)(3)(4)(5)(6)(7)(8)(9)(10)(11)(12)(13)(14)(15) \\
& \alpha_{1}=\left(\begin{array}{lllllllllllllll}
1 & 3 & 9 & 10 & 13 & 8 & 7 & 4 & 12 & 5 & 15 & 14 & 11 & 2 & 6
\end{array}\right)
\end{aligned}
$$




$$
\begin{aligned}
& \alpha_{2}=\left(\begin{array}{lllllllllllllll}
1 & 9 & 13 & 7 & 12 & 15 & 11 & 6 & 3 & 10 & 8 & 4 & 5 & 14 & 2
\end{array}\right) \\
& \alpha_{3}=\left(\begin{array}{lll}
1 & 9 & 6
\end{array}\right)\left(\begin{array}{lllllll}
2 & 5 & 7 & 14 & 15 & 11 & 10
\end{array}\right)\left(\begin{array}{ll}
3 & 12
\end{array}\right)\left(\begin{array}{ll}
4 & 8
\end{array}\right)\left(\begin{array}{l}
13
\end{array}\right) \\
& \alpha_{4}=\left(\begin{array}{llllll}
1 & 9 & 10 & 15 & 11 & 12
\end{array}\right)\left(\begin{array}{lll}
2 & 8 & 4
\end{array}\right)\left(\begin{array}{ll}
3 & 14
\end{array}\right)\left(\begin{array}{lll}
5 & 13 & 6
\end{array}\right)\left(\begin{array}{l}
7
\end{array}\right) \\
& \alpha_{5}=\left(\begin{array}{lllllllllll}
1 & 9 & 12 & 7 & 5 & 6 & 8 & 4 & 15 & 11 & 14
\end{array}\right)\left(\begin{array}{l}
2
\end{array}\right)\left(\begin{array}{llll}
3 & 7 & 13 & 10
\end{array}\right) \\
& \alpha_{6}=\left(\begin{array}{lllll}
1 & 9 & 14 & 5 & 10
\end{array}\right)\left(\begin{array}{lllll}
2 & 15 & 11 & 7 & 6
\end{array}\right)\left(\begin{array}{lllll}
3 & 13 & 12 & 8 & 4
\end{array}\right) \\
& \alpha_{7}=\left(\begin{array}{lllllllllllllll}
1 & 9 & 7 & 10 & 5 & 12 & 2 & 3 & 6 & 15 & 11 & 13 & 14 & 8 & 4
\end{array}\right)
\end{aligned}
$$

\section{Algebraic Structure of the $\bigcup_{x_{k}}^{n}$-Permutations}

Here, we consider the $\bigcup_{x_{k}}^{n}$-permutations with $t=m$, where $m$ is the middle position among the $n$ positions in set $N$, and $n \in \mathbb{N}$ is a product of two primes. In the next result, we prove that this category of permutations form a group under permutation multiplication.

Theorem 5.1 Let $n \in \mathbb{N}$ be a product of two primes and let $N=\left\{x_{1}, x_{2}, \ldots, x_{n}\right\}$. Let $m$ be the element in the middle position of set $N$. Then for $t=m$, the set $N_{x_{m}}^{n}$ of all the $\bigcup_{x_{m}}^{n}$-permutations on $N$ is a group under permutation multiplication. This group is abelian.

\section{Proof}

Clearly, $N_{x_{m}}^{n}$ is closed under permutation multiplication for if $\alpha_{i}, \alpha_{j} \in N_{x_{m}}^{n}$, then $\alpha_{i} \alpha_{j}$ is another permutation in $N_{x_{m}}^{n}$.

Next, $N_{x_{m}}^{n}$ is associative under permutation multiplication for if $\alpha_{i}, \alpha_{j}, \alpha_{k} \in N_{x_{m}}^{n}$, then $\alpha_{i}\left(\alpha_{j} \alpha_{k}\right)=\left(\alpha_{i} \alpha_{j}\right) \alpha_{k}$

Also, the permutation $\alpha_{0} \in N_{x_{m}}^{n}$ and is the identity permutation in $N_{x_{m}}^{n}$.

Finally, for every $\alpha_{i} \in N_{x_{m}}^{n}$, there must be a permutation $\alpha_{i}{ }^{\prime} \in N_{x_{m}}^{n}$ with $\alpha_{i} \alpha_{i}{ }^{\prime}=\alpha_{0}$. That is, every permutation in $N_{x_{m}}^{n}$ has an inverse in $N_{x_{m}}^{n}$. Thus, $N_{x_{m}}^{n}$ is a group. This group is abelian for we have $\alpha_{i} \alpha_{j}=\alpha_{j} \alpha_{i} \forall \alpha_{i}, \alpha_{j} \in N_{x_{m}}^{n}$.

Corollary 5.2 The group $N_{x_{m}}^{n}$ is a subgroup of the group $N_{n}$ of all permutations on $N$.

\section{Proof}

We have seen in theorem 2.2 that $N_{n}$ is a group under permutation multiplication. we have seen from corollary 4.3 that $N_{x_{m}}^{n}$ is a subset of $N_{n}$, the set of all permutations on $N$. Also, we have seen in theorem 5.1 that $N_{x_{m}}^{n}$ is a group. Thus, we conclude that $N_{x_{m}}^{n}$ is a subgroup of $N_{n}$.

Three categories of permutations were considered for this category, that is, when $n=9$, $n=15$ and $n=21$. When $q=3, m=5$ and $n=9$, then, $x_{m}=x_{5}=5$. In this case we have: 


$$
\alpha_{0}=\left(\begin{array}{lllllllll}
1 & 2 & 3 & 4 & 5 & 6 & 7 & 8 & 9 \\
1 & 2 & 3 & 4 & 5 & 6 & 7 & 8 & 9
\end{array}\right)
$$

When $N$ is mapped the first time, the first permutation denoted by $\alpha_{1}$ is obtained as shown below;

$$
\begin{aligned}
& \alpha_{0}=\left(\begin{array}{lllllllll}
1 & 2 & 3 & 4 & 5 & 6 & 7 & 8 & 9
\end{array}\right) \\
& C_{1}=\{1,4,7\}, C_{2}=\{2,5,8\}, C_{3}=\{3,6,9\}
\end{aligned}
$$

Then,

$$
P_{\alpha_{0}}^{9}=\{\{1,4,7\},\{2,5,8\},\{3,6,9\}\}
$$

Taking the union, we obtain

$$
\bigcup_{5}^{9}\left[P_{\alpha_{0}}^{9}\right]=\{1,4,7,2,5,8,3,6,9\}
$$

Therefore

$$
\alpha_{1}=\left(\begin{array}{lllllllll}
1 & 2 & 3 & 4 & 5 & 6 & 7 & 8 & 9 \\
1 & 4 & 7 & 2 & 5 & 8 & 3 & 6 & 9
\end{array}\right)
$$

The set $N_{5}^{9}=\left\{\alpha_{0}, \alpha_{1}\right\}$ is a group whose group table is

TABLE 2. $N_{5}^{9}$ Group Table

\begin{tabular}{c|cc}
$\circ$ & $\alpha_{0}$ & $\alpha_{1}$ \\
\hline$\alpha_{0}$ & $\alpha_{0}$ & $\alpha_{1}$ \\
$\alpha_{1}$ & $\alpha_{1}$ & $\alpha_{0}$
\end{tabular}

Note that we have:

$$
\begin{aligned}
\alpha_{1} \alpha_{1} & =\left(\begin{array}{lllllllll}
1 & 2 & 3 & 4 & 5 & 6 & 7 & 8 & 9 \\
1 & 4 & 7 & 2 & 5 & 8 & 3 & 6 & 9
\end{array}\right)\left(\begin{array}{lllllllll}
1 & 2 & 3 & 4 & 5 & 6 & 7 & 8 & 9 \\
1 & 4 & 7 & 2 & 5 & 8 & 3 & 6 & 9
\end{array}\right) \\
& =\left(\begin{array}{lllllllll}
1 & 2 & 3 & 4 & 5 & 6 & 7 & 8 & 9 \\
1 & 2 & 3 & 4 & 5 & 6 & 7 & 8 & 9
\end{array}\right) \\
& =\alpha_{0}
\end{aligned}
$$

Clearly, this group is isomorphic to $\left(\mathbb{Z}_{2},+\right)$, for if we define a function $\Phi: N_{5}^{9} \rightarrow \mathbb{Z}_{2}$ by $\Phi\left(\alpha_{i}\right)=i$ for some $i=0,1$, then $\Phi$ is clearly an isomorphism for we have $\Phi\left(\alpha_{0}\right)=0$ and $\Phi\left(\alpha_{1}\right)=1$. Note that the cayley table for $\left(\mathbb{Z}_{2},+\right)$ is given by:

TABLE 3. Table for $\left(\mathbb{Z}_{2},+\right)$

\begin{tabular}{c|cc}
+ & 0 & 1 \\
\hline 0 & 0 & 1 \\
1 & 1 & 0
\end{tabular}


When we consider $m=8, p=3, x_{m}=m_{8}=8$ and $n=15$, we obtain

$$
N=\{1,2,3,4,5,6,7,8,9,10,11,12,13,14,15\}
$$

Therefore,

$$
\alpha_{0}=\left(\begin{array}{lllllllllllllll}
1 & 2 & 3 & 4 & 5 & 6 & 7 & 8 & 9 & 10 & 11 & 12 & 13 & 14 & 15 \\
1 & 2 & 3 & 4 & 5 & 6 & 7 & 8 & 9 & 10 & 11 & 12 & 13 & 14 & 15
\end{array}\right)
$$

$N$ undergoes the first permutation, we obtain the permutation $\alpha_{1}$ as shown below.

$$
\begin{aligned}
\alpha_{0} & =\left(\begin{array}{lllllllllllllll}
1 & 2 & 3 & 4 & 5 & 6 & 7 & 8 & 9 & 10 & 11 & 12 & 13 & 14 & 15
\end{array}\right) \\
C_{1} & =\{1,4,7,10,13\}, C_{2}=\{2,5,8,11,14\}, C_{3}=\{3,6,9,12,15\}
\end{aligned}
$$

Then,

$$
P_{\alpha_{0}}^{15}=\{\{1,4,7,10,13\},\{2,5,8,11,14\},\{3,6,9,12,15\}\}
$$

Taking the union,we obtain

$$
\bigcup_{8}^{15}\left[P_{\alpha_{0}}^{15}\right]=\{1,4,7,10,13,2,5,8,11,14,3,6,9,12,15\}
$$

\section{Therefore}

$$
\alpha_{1}=\left(\begin{array}{ccccccccccccccc}
1 & 2 & 3 & 4 & 5 & 6 & 7 & 8 & 9 & 10 & 11 & 12 & 13 & 14 & 15 \\
1 & 4 & 7 & 10 & 13 & 2 & 5 & 8 & 11 & 14 & 3 & 6 & 9 & 12 & 15
\end{array}\right)
$$

When $N$ undergoes the next permutation we obtain $\alpha_{2}$ as shown below;

$$
\begin{aligned}
& \alpha_{1}=\left(\begin{array}{lllllllllllllll}
1 & 4 & 7 & 10 & 13 & 2 & 5 & 8 & 11 & 14 & 3 & 6 & 9 & 12 & 15
\end{array}\right) \\
& C_{1}=\{1,10,5,14,9\}, C_{2}=\{4,13,8,3,12\}, C_{3}=\{7,2,11,6,15\}
\end{aligned}
$$

Then,

$$
P_{\alpha_{1}}^{15}=\{\{1,10,5,14,9\},\{4,13,8,3,12\},\{7,2,11,6,15\}\}
$$

Taking the union,we obtain

$$
\bigcup_{8}^{15}\left[P_{\alpha_{1}}^{15}\right]=\{1,10,5,14,9,4,13,8,3,12,7,2,11,6,15\}
$$

Therefore

$$
\alpha_{2}=\left(\begin{array}{ccccccccccccccc}
1 & 2 & 3 & 4 & 5 & 6 & 7 & 8 & 9 & 10 & 11 & 12 & 13 & 14 & 15 \\
1 & 10 & 5 & 14 & 9 & 4 & 13 & 8 & 3 & 12 & 7 & 2 & 11 & 6 & 15
\end{array}\right)
$$

$N$ undergoes the next permutation and we obtain $\alpha_{3}$ as shown below;

$$
\begin{aligned}
& \alpha_{2}=\left(\begin{array}{lllllllllllllll}
1 & 10 & 5 & 14 & 9 & 4 & 13 & 8 & 3 & 12 & 7 & 2 & 11 & 6 & 15
\end{array}\right) \mathrm{g} \\
& C_{1}=\{1,14,13,12,11\}, C_{2}=\{10,9,8,7,6\}, C_{3}=\{5,4,3,2,15\}
\end{aligned}
$$

Then,

$$
P_{\alpha_{2}}^{15}=\{\{1,14,13,12,11\},\{10,9,8,7,6\},\{5,4,3,2,15\}\}
$$

Taking the union,we obtain

$$
\bigcup_{8}^{15}\left[P_{\alpha_{2}}^{15}\right]=\{1,14,13,12,11,10,19,8,7,6,5,4,3,2,15\}
$$

Therefore 


$$
\alpha_{3}=\left(\begin{array}{ccccccccccccccc}
1 & 2 & 3 & 4 & 5 & 6 & 7 & 8 & 9 & 10 & 11 & 12 & 13 & 14 & 15 \\
1 & 14 & 13 & 12 & 11 & 10 & 9 & 8 & 7 & 6 & 5 & 4 & 3 & 2 & 15
\end{array}\right)
$$

The fourth permutation denoted by $\alpha_{4}$ is obtained when $N$ undergoes the next permutation as shown below.

$$
\begin{aligned}
& \alpha_{3}=\left(\begin{array}{lllllllllllllll}
1 & 14 & 13 & 12 & 11 & 10 & 9 & 8 & 7 & 6 & 5 & 4 & 3 & 2 & 15
\end{array}\right) \\
& C_{1}=\{1,12,9,6,3\}, C_{2}=\{14,11,8,5,2\}, C_{3}=\{13,10,7,4,15\}
\end{aligned}
$$

Then,

$$
P_{\alpha_{3}}^{15}=\{\{1,12,9,6,3\},\{14,11,8,5,2\},\{13,10,7,4,15\}\}
$$

Taking the union,we obtain

$$
\bigcup_{8}^{15}\left[P_{\alpha_{3}}^{15}\right]=\{1,12,9,6,3,14,11,8,5,2,13,10,7,4,15\}
$$

Therefore

$$
\alpha_{4}=\left(\begin{array}{ccccccccccccccc}
1 & 2 & 3 & 4 & 5 & 6 & 7 & 8 & 9 & 10 & 11 & 12 & 13 & 14 & 15 \\
1 & 12 & 9 & 6 & 3 & 14 & 11 & 8 & 5 & 2 & 13 & 10 & 7 & 4 & 15
\end{array}\right)
$$

When $N$ goes through the fifth permutation we obtain $\alpha_{5}$ as shown below;

$$
\begin{aligned}
& \alpha_{4}=\left(\begin{array}{lllllllllllllll}
1 & 12 & 9 & 6 & 3 & 14 & 11 & 8 & 5 & 2 & 13 & 10 & 7 & 4 & 15
\end{array}\right) \\
& C_{1}=\{1,6,11,2,7\}, C_{2}=\{12,3,8,13,4\}, C_{3}=\{9,14,5,10,15\}
\end{aligned}
$$

Then,

$$
P_{\alpha_{4}}^{15}=\{\{1,6,11,2,7\},\{12,3,8,13,4\},\{9,14,5,10,15\}\}
$$

Taking the union,we obtain

$$
\bigcup_{8}^{15}\left[P_{\alpha_{4}}^{15}\right]=\{1,6,11,2,7,12,3,8,13,4,9,14,5,10,15\}
$$

Therefore

$$
\alpha_{5}=\left(\begin{array}{ccccccccccccccc}
1 & 2 & 3 & 4 & 5 & 6 & 7 & 8 & 9 & 10 & 11 & 12 & 13 & 14 & 15 \\
1 & 6 & 11 & 2 & 7 & 12 & 3 & 8 & 13 & 4 & 9 & 14 & 5 & 10 & 15
\end{array}\right)
$$

The set $N_{8}^{15}=\left\{\alpha_{0}, \alpha_{1}, \alpha_{2}, \alpha_{3}, \alpha_{4}, \alpha_{5}\right\}$ is a group whose cayley table is as shown by Table 4 below:

Clearly, this group is isomorphic to $\left(\mathbb{Z}_{6},+\right)$, for if we define a function $\Phi: N_{8}^{15} \rightarrow \mathbb{Z}_{6}$ by $\Phi\left(\alpha_{i}\right)=i$ for some $i=0,1,2,3,4,5$, then $\Phi$ is clearly an isomorphism for we have $\Phi\left(\alpha_{0}\right)=0$, $\Phi\left(\alpha_{1}\right)=1 \Phi\left(\alpha_{2}\right)=2, \Phi\left(\alpha_{3}\right)=3, \Phi\left(\alpha_{4}\right)=4$ and $\Phi\left(\alpha_{5}\right)=5$. Note that the cayley table for $\left(\mathbb{Z}_{6},+\right)$ is as shown in Table 5 below:

When $m=11, q=3$ and $n=21$, we obtain

$$
N=\{1,2,3,4,5,6,7,8,9,10,11,12,13,14,15,16,17,18,19,20,21\}
$$

Therefore, 
TABLE 4. $N_{8}^{15}$ Group Table

\begin{tabular}{c|cccccc}
$\circ$ & $\alpha_{0}$ & $\alpha_{1}$ & $\alpha_{2}$ & $\alpha_{3}$ & $\alpha_{4}$ & $\alpha_{5}$ \\
\hline$\alpha_{0}$ & $\alpha_{0}$ & $\alpha_{1}$ & $\alpha_{2}$ & $\alpha_{3}$ & $\alpha_{4}$ & $\alpha_{5}$ \\
$\alpha_{1}$ & $\alpha_{1}$ & $\alpha_{2}$ & $\alpha_{3}$ & $\alpha_{4}$ & $\alpha_{5}$ & $\alpha_{0}$ \\
$\alpha_{2}$ & $\alpha_{2}$ & $\alpha_{3}$ & $\alpha_{4}$ & $\alpha_{5}$ & $\alpha_{0}$ & $\alpha_{1}$ \\
$\alpha_{3}$ & $\alpha_{3}$ & $\alpha_{4}$ & $\alpha_{5}$ & $\alpha_{0}$ & $\alpha_{1}$ & $\alpha_{2}$ \\
$\alpha_{4}$ & $\alpha_{4}$ & $\alpha_{5}$ & $\alpha_{0}$ & $\alpha_{1}$ & $\alpha_{2}$ & $\alpha_{3}$ \\
$\alpha_{5}$ & $\alpha_{5}$ & $\alpha_{0}$ & $\alpha_{1}$ & $\alpha_{2}$ & $\alpha_{3}$ & $\alpha_{4}$
\end{tabular}

TABLE 5. $\left(\mathbb{Z}_{6},+\right)$

\begin{tabular}{c|llllll}
+ & 0 & 1 & 2 & 3 & 4 & 5 \\
\hline 0 & 0 & 1 & 2 & 3 & 4 & 5 \\
1 & 1 & 2 & 3 & 4 & 5 & 0 \\
2 & 2 & 3 & 4 & 5 & 0 & 1 \\
3 & 3 & 4 & 5 & 0 & 1 & 2 \\
4 & 4 & 5 & 0 & 1 & 2 & 3 \\
5 & 5 & 0 & 1 & 2 & 3 & 4
\end{tabular}

$\alpha_{0}=\left(\begin{array}{lllllllllllllllllllll}1 & 2 & 3 & 4 & 5 & 6 & 7 & 8 & 9 & 10 & 11 & 12 & 13 & 14 & 15 & 16 & 17 & 18 & 19 & 20 & 21 \\ 1 & 2 & 3 & 4 & 5 & 6 & 7 & 8 & 9 & 10 & 11 & 12 & 13 & 14 & 15 & 16 & 17 & 18 & 19 & 20 & 21\end{array}\right)$ $N$ undergoes a permutation we obtain $\alpha_{1}$ as shown below.

$$
\begin{aligned}
& \alpha_{0}=\left(\begin{array}{lllllllllllllllllllll}
1 & 2 & 3 & 4 & 5 & 6 & 7 & 8 & 9 & 10 & 11 & 12 & 13 & 14 & 15 & 16 & 17 & 18 & 19 & 20 & 21
\end{array}\right) \\
& C_{1}=\{1,4,7,10,13,11,16,19\}, C_{2}=\{2,5,8,11,14,17,20\}, C_{3}=\{3,6,9,12,15,18,21\}
\end{aligned}
$$

Then,

$$
P_{\alpha_{0}}^{21}=\{\{1,4,7,10,13,11,16,19\},\{2,5,8,11,14,17,20\},\{3,6,9,12,15,18,21\}\}
$$

Taking the union,we obtain

$$
\bigcup_{11}^{21}\left[P_{\alpha_{0}}^{21}\right]=\{1,4,7,10,13,11,16,19,2,5,8,11,14,17,20,3,6,9,12,15,18,21\}
$$

Therefore

$$
\alpha_{1}=\left(\begin{array}{ccccccccccccccccccccc}
1 & 2 & 3 & 4 & 5 & 6 & 7 & 8 & 9 & 10 & 11 & 12 & 13 & 14 & 15 & 16 & 17 & 18 & 19 & 20 & 21 \\
1 & 4 & 7 & 10 & 13 & 16 & 19 & 2 & 5 & 8 & 11 & 14 & 17 & 20 & 3 & 6 & 9 & 12 & 15 & 18 & 21
\end{array}\right)
$$

When $N$ undergoes the next permutation we obtain $\alpha_{2}$ as shown below;

$$
\alpha_{1}=\left(\begin{array}{lllllllllllllllllllll}
1 & 4 & 7 & 10 & 13 & 16 & 19 & 2 & 5 & 8 & 11 & 14 & 17 & 20 & 3 & 6 & 9 & 12 & 15 & 18 & 21
\end{array}\right)
$$




$$
C_{1}=\{1,10,19,8,17,6,15\}, C_{2}=\{4,13,2,11,20,9,18\}, C_{3}=\{7,16,5,14,3,12,21\}
$$

Then,

$$
P_{\alpha_{1}}^{21}=\{\{1,10,19,8,17,6,15\},\{4,13,2,11,20,9,18\},\{7,16,5,14,3,12,21\}\}
$$

Taking the union,we obtain $\bigcup_{11}^{21}\left[P_{\alpha_{1}}^{21}\right]=\{1,10,19,8,17,6,15,4,13,2,11,20,9,18,7,16,5,14,3,12,21\}$

Therefore

$\alpha_{2}=\left(\begin{array}{ccccccccccccccccccccc}1 & 2 & 3 & 4 & 5 & 6 & 7 & 8 & 9 & 10 & 11 & 12 & 13 & 14 & 15 & 16 & 17 & 18 & 19 & 20 & 21 \\ 1 & 10 & 19 & 8 & 17 & 6 & 15 & 4 & 13 & 2 & 11 & 20 & 9 & 18 & 7 & 16 & 5 & 14 & 3 & 12 & 21\end{array}\right)$

When $N$ undergoes the next permutation we obtain $\alpha_{3}$ as shown below;

$$
\begin{aligned}
& \alpha_{2}=\left(\begin{array}{lllllllllllllllllllll}
1 & 10 & 19 & 8 & 17 & 6 & 15 & 4 & 13 & 2 & 11 & 20 & 9 & 18 & 7 & 16 & 5 & 14 & 3 & 12 & 21
\end{array}\right) \\
& C_{1}=\{1,8,15,2,9,16,3\}, C_{2}=\{10,17,4,11,18,5,12\}, C_{3}=\{19,6,13,20,7,14,21\}
\end{aligned}
$$

Then,

$$
P_{\alpha_{2}}^{21}=\{\{1,8,15,2,9,16,3\},\{10,17,4,11,18,5,12\},\{19,6,13,20,7,14,21\}\}
$$

Taking the union, we obtain

$$
\bigcup_{11}^{21}\left[P_{\alpha_{2}}^{21}\right]=\{1,8,15,2,9,16,3,10,17,4,11,18,5,12,19,6,13,20,7,14,21\}
$$

Therefore

$\alpha_{3}=\left(\begin{array}{ccccccccccccccccccccc}1 & 2 & 3 & 4 & 5 & 6 & 7 & 8 & 9 & 10 & 11 & 12 & 13 & 14 & 15 & 16 & 17 & 18 & 19 & 20 & 21 \\ 1 & 8 & 15 & 2 & 9 & 16 & 3 & 10 & 17 & 4 & 11 & 18 & 5 & 12 & 19 & 6 & 13 & 20 & 7 & 14 & 21\end{array}\right)$

The set $N_{11}^{21}=\left\{\alpha_{0}, \alpha_{1}, \alpha_{2}, \alpha_{3}\right\}$ is a group whose group is shown below:

TABle 6. $N_{11}^{21}$ Group Table

\begin{tabular}{c|cccc}
$\circ$ & $\alpha_{0}$ & $\alpha_{1}$ & $\alpha_{2}$ & $\alpha_{3}$ \\
\hline$\alpha_{0}$ & $\alpha_{0}$ & $\alpha_{1}$ & $\alpha_{2}$ & $\alpha_{3}$ \\
$\alpha_{1}$ & $\alpha_{1}$ & $\alpha_{2}$ & $\alpha_{3}$ & $\alpha_{0}$ \\
$\alpha_{2}$ & $\alpha_{2}$ & $\alpha_{3}$ & $\alpha_{0}$ & $\alpha_{1}$ \\
$\alpha_{3}$ & $\alpha_{3}$ & $\alpha_{0}$ & $\alpha_{1}$ & $\alpha_{2}$
\end{tabular}

Clearly,this group is isomorphic to $\left(\mathbb{Z}_{4},+\right)$.

For if we define a function $\Phi: N_{11}^{21} \rightarrow \mathbb{Z}_{4}$ by $\Phi\left(\alpha_{i}=i\right)$ for some $i=0,1,2,3$, then $\Phi$ is clearly an isomorphism for we have $\Phi\left(\alpha_{0}\right)=0, \Phi\left(\alpha_{1}\right)=1, \Phi\left(\alpha_{2}\right)=2$ and $\Phi\left(\alpha_{3}\right)=3$. Note that the cayley table for $\left(\mathbb{Z}_{4},+\right)$ is as shown below:

In this section, we have considered a class of permutations forming a group. Three categories are considered and their cycles are provided below.

When $n=9$, there are two permutations. Their cycles are given below: 


\begin{tabular}{|c|c|c|c|c|}
\hline+ & 0 & 1 & 2 & 3 \\
\hline 0 & 0 & 1 & 2 & 3 \\
\hline 1 & 1 & 2 & 3 & 0 \\
\hline 2 & 2 & 3 & 0 & 1 \\
\hline 3 & 3 & 0 & 1 & 2 \\
\hline
\end{tabular}

$$
\begin{aligned}
& \alpha_{0}=(1)(2)(3)(4)(5)(6)(7)(8)(9) \\
& \alpha_{1}=\left(\begin{array}{l}
1
\end{array}\right)\left(\begin{array}{ll}
2 & 4
\end{array}\right)\left(\begin{array}{ll}
3 & 7
\end{array}\right)\left(\begin{array}{l}
5
\end{array}\right)\left(\begin{array}{ll}
6 & 8
\end{array}\right)\left(\begin{array}{l}
9
\end{array}\right)
\end{aligned}
$$

When $n=15$, there are six permutations and their cycles are shown below:

$$
\begin{aligned}
& \alpha_{0}=(1)(2)(3)(4)(5)(6)(7)(8)(9)(10)(11)(12)(13)(14)(15) \\
& \alpha_{1}=\left(\begin{array}{l}
1
\end{array}\right)\left(\begin{array}{llllll}
2 & 4 & 10 & 14 & 12 & 6
\end{array}\right)\left(\begin{array}{llllll}
3 & 7 & 5 & 13 & 9 & 11
\end{array}\right)(8)\left(\begin{array}{l}
15
\end{array}\right) \\
& \alpha_{2}=\left(\begin{array}{l}
1
\end{array}\right)\left(\begin{array}{lll}
2 & 10 & 12
\end{array}\right)\left(\begin{array}{lll}
3 & 5 & 9
\end{array}\right)\left(\begin{array}{lll}
4 & 14 & 6
\end{array}\right)\left(\begin{array}{lll}
7 & 13 & 11
\end{array}\right)(8)\left(\begin{array}{l}
15
\end{array}\right) \\
& \alpha_{3}=\left(\begin{array}{l}
1
\end{array}\right)\left(\begin{array}{ll}
2 & 14
\end{array}\right)\left(\begin{array}{ll}
3 & 13
\end{array}\right)\left(\begin{array}{ll}
4 & 12
\end{array}\right)\left(\begin{array}{ll}
5 & 11
\end{array}\right)\left(\begin{array}{ll}
6 & 10
\end{array}\right)\left(\begin{array}{ll}
7 & 9
\end{array}\right)\left(\begin{array}{l}
8
\end{array}\right)\left(\begin{array}{l}
15
\end{array}\right) \\
& \alpha_{4}=\left(\begin{array}{l}
1
\end{array}\right)\left(\begin{array}{lll}
2 & 10 & 12
\end{array}\right)\left(\begin{array}{lll}
3 & 5 & 9
\end{array}\right)\left(\begin{array}{lll}
4 & 14 & 6
\end{array}\right)\left(\begin{array}{lll}
7 & 13 & 11
\end{array}\right)(8)\left(\begin{array}{l}
15
\end{array}\right) \\
& \alpha_{5}=\left(\begin{array}{l}
1
\end{array}\right)\left(\begin{array}{llllll}
2 & 6 & 12 & 14 & 10 & 4
\end{array}\right)\left(\begin{array}{llllll}
3 & 11 & 9 & 13 & 5 & 7
\end{array}\right)(8)\left(\begin{array}{l}
15
\end{array}\right)
\end{aligned}
$$

When $n=21$, there are four permutations and their cycles are shown below:

$$
\begin{aligned}
& \alpha_{0}=(1)(2)(3)(4)(5)(6)(7)(8)(9)(10)(11)(12)(13)(14)(15)(16)(17)(18)(19)(20)(21) \\
& \alpha_{1}=\left(\begin{array}{l}
1
\end{array}\right)\left(\begin{array}{llll}
2 & 4 & 10 & 8
\end{array}\right)\left(\begin{array}{llll}
3 & 7 & 19 & 15
\end{array}\right)\left(\begin{array}{llll}
5 & 13 & 17 & 9
\end{array}\right)\left(\begin{array}{ll}
6 & 16
\end{array}\right)\left(\begin{array}{l}
11
\end{array}\right) \\
& \left(\begin{array}{llll}
12 & 14 & 20 & 18
\end{array}\right)(21) \\
& \alpha_{2}=\left(\begin{array}{l}
1
\end{array}\right)\left(\begin{array}{ll}
1 & 10
\end{array}\right)\left(\begin{array}{ll}
3 & 19
\end{array}\right)\left(\begin{array}{ll}
4 & 8
\end{array}\right)\left(\begin{array}{ll}
5 & 17
\end{array}\right)\left(\begin{array}{l}
6
\end{array}\right)\left(\begin{array}{ll}
7 & 15
\end{array}\right)\left(\begin{array}{ll}
9 & 13
\end{array}\right)\left(\begin{array}{l}
11
\end{array}\right) \\
& \left(\begin{array}{ll}
12 & 20
\end{array}\right)\left(\begin{array}{ll}
14 & 18
\end{array}\right)(16)(21) \\
& \alpha_{3}=\left(\begin{array}{l}
1
\end{array}\right)\left(\begin{array}{llll}
2 & 8 & 10 & 4
\end{array}\right)\left(\begin{array}{llll}
3 & 15 & 19 & 7
\end{array}\right)\left(\begin{array}{llll}
5 & 9 & 17 & 13
\end{array}\right)\left(\begin{array}{ll}
6 & 16
\end{array}\right)\left(\begin{array}{l}
11
\end{array}\right) \\
& \left(\begin{array}{llll}
7 & 18 & 20 & 14
\end{array}\right)\left(\begin{array}{l}
21
\end{array}\right)
\end{aligned}
$$

\section{Conclusions and Recommendations}

In sections 4 and 5, two classes of permutations are generated. In section 4, examples of permutations not forming a group are presented while section 5 presents a class of permutations forming a group. The generated permutations have also been presented in terms of their cycles. It would be interesting to further analyze these permutations with an aim of determining more properties like the nature of their signatures among other properties. A study of the graphs resulting from these permutations may also reveal more of their properties. 


\section{REFERENCES}

[1] J. B. Fraleigh, A First Course in Abstract Algebra. 7th Edition, Addison Wesley Publishing Company, Boston, 1982.

[2] D. Roulants and F. Ruskey, Generating Permutations With Given Ups and Downs, Discrete Appl. Math. 36(1)(1992), 57-65.

[3] G. Sypro, The Number of Permutations With a Given Signiture and the Expectations of their Elements, Discrete Math. 226 (2001), 423-430.

[4] H. O. Foulkes, Enumeration of Permutations with Prescribed Up-Down and Inversions Sequences, Discrete Math. 15 (1970), 235-252.

[5] N. A. Dershowitz, A Simplified Loot Free Algorithm for Generating Permutations, BIT. 5 (1975), 158-164.

[6] F. A. Breuti, A class of q-Symmetric Functions Arising From Plethysm, J. Comb. 19 (2000), 137-170.

[7] J. Rammel and M. Riehl, Generating Functions for Permutations Which Contain a Given Descent Set, Electron. J. Comb. 17 (2010), R27.

[8] V. A. Vajnovszki, Loopless Algorithm for Generating the Permutations of a Multiset, Theor. Comput. Sci. 307 (2003), 415-431.

[9] J. N. Kwak and J. Lee, Isomorphism Classes of Cycle Permutation Graphs, Discrete Math. 105 (1992), 131-142.

[10] F. Ruskey, Transposition Generation of Alternating Permutations, Order. 6 (1989), 227-233. 